\title{
Fibrosis Quística Pulmonar, Gástrica y Gastro-Pulmonar en la Actualidad: Una Revisión Sistémica
}

\section{Cystic Fibrosis Pulmonary Gastric and Gastropulmonar Today: Systemic Review}

\author{
Bocanegra Amaya $C^{1}$, Bula Muñoz $\mathrm{LC}^{2}$ \\ ${ }^{1}$ Programa de Odontología - Universidad del Magdalena, ${ }^{2}$ Programa de Medicina - Universidad del Magdalena
}

\begin{abstract}
Resumen
La Fibrosis Quística (FQ) es una enfermedad que produce compromiso multisistémico, crónico y en muchos casos, progresivo. Más de 20 años han transcurrido desde la identificación del gen CFTR, cuyas mutaciones la producen. Desde entonces, han ocurrido importantes avances en la comprensión de su patogenia, muchos de los cuales se han traducido en nuevas estrategias terapéuticas y mejorías en la sobrevida y calidad de vida de los pacientes. Sin embargo, mucho nos queda aún por entender y por avanzar en tratamiento y resultados. Las pruebas para diagnosticarla consisten principalmente en la prueba del sudor, respaldada con pruebas de ADN.Varios estudios clínicos indican que el estado nutricional desempeña un papel importante en la progresión de la afección pulmonar. Estudios longitudinales apuntan a una ventaja de supervivencia para los pacientes con un status nutricional óptimo, es por eso que la alimentación juega un papel crucial en la calidad de vida de las personas que la padecen.
\end{abstract}

Palabras Claves: CFTR, fibrosis quística, $\triangle F Q$ 508, nutrición (Fuente: DeCS).

\begin{abstract}
Cystic Fibrosis ( CF) is a multisystem disease that causes chronic and in many cases, progressive. More than 20 years have elapsed since the identification of the CFTR gene, whose mutations cause the disease. Since then, there have been significant advances in understanding the pathogenesis of the disease, many of which have led to new therapeutic strategies and improvements in survival and quality of life of patients. However, much we have yet to understand and to advance treatment and outcomes. Tests to diagnose this disease consist primarily sweat test, tests DNA. Many backed clinical studies indicate that nutritional status plays an important role in the progression of lung disease. Longitudinal studies suggest a survival advantage for patients with optimal nutritional status, is why nutrition plays a crucial role in the quality of life of those who suffer.
\end{abstract}

Keywords: CFTR, cysticfibrosis, $\triangle F Q$ 508, nutrition (Source: MeSH, NLM).

Correspondencia: Carmen Bocanegra Amaya. Facultad de Medicina. Universidad del magdalena. Santa Marta, Magdalena, Colombia. carboama@gmail.com.

Citar: Bocanegra Amaya C, Bula Muñoz L. Fibrosis Quística Pulmonar, Gástrica y Gastro-Pulmonar en la Actualidad: Una Revisión Sistémica. Cienc e Innovación en Salud [Internet]. 2015 Jan 1;3(1):1-1. Available from: http://publicaciones.unisimonbolivar.edu.co:82/rdigital/ojs/index.php/innovacionsalud/article /view/238/236.

Parte de este trabajo se encuentran soportado en la investigación titulada «Diagnóstico del Nivel de Cumplimiento de Condiciones Esenciales de Servicio Farmacéutico en Empresas Sociales del Estado, Acorde con la Normatividad Vigente - 2008» tesis para obtener el título de Máster Internacional en Atención Farmacéutica de la Universidad de Granada (España).

Recibido: Oct. 12 de 2014 / Modificado: Nov. 11 de 2014 / Aceptado: Nov. 21 de 2014.

\section{INTRODUCCIÓN}

La fibrosis quística es la enfermedad autosómica recesiva más frecuente en poblaciones caucásicas que afecta las características químicas. y físicas en las secreciones producidas por diversas glándulas del organismo, incluidas las bronquiales, intestinales, pancreáticas, sudoríparas, hepáticas, del sistema reproductor $\mathrm{u}$ otras. El espesamiento de dichas secreciones obstruye progresivamente los canalículos excretores hasta ocasionar una disfunción de las glándulas y del órgano afectado (1). El gen regulador de la conductancia transmembranal de la fibrosis quística (ctfr) fue clonado en 1989 e identificada la mutación principal $\triangle F Q 508$ (2). Desde entonces más de 1400 mutaciones diferentes en el gen cftr han sido descritas (3), existe una mutación mayoritaria es la delta F508 localizado en el cromosoma 7, presente en un promedio del $70 \%$ a $80 \%$ de los alelos de $F Q$ analizados en el continente americano y un $67 \%$ de los alelos de FQ analizados a nivel mundial (4).

Hasta hace poco tiempo, existía la creencia errada en los profesionales de la salud que esta entidad era rara en los países latinoamericanos; lo que lamentablemente condujo a una tardanza en su reconocimiento, diagnóstico y cuidado inadecuado. Gracias a la presión inicial y continua de familias de pacientes afectados por esta condición y también del interés y conocimientos de varios especialistas respiratorios pediátricos, el pronóstico de ésta enfermedad en nuestros países ha comenzado a mejorar, pero lastimosamente la mayor parte de los pacientes son niños y su calidad de vida es regular a mala. Existen varias razones para esto: recursos limitados, falta de sospecha clínica, falta de facilidades de laboratorio diagnóstico adecuado (test del sudor, estudio genético) y falta de entrenamiento y práctica adecuada del personal médico. 
Enfocándonos en la enfermedad, resulta que las principales anormalidades están dadas por: Concentración anormal de iones inorgánicos en las secreciones glandulares serosas, que se refleja en un aumento de cloruro de sodio en sudor; aumento en la viscosidad de las secreciones glandulares mucosas que conduce a obstrucción de los canales excretores y pérdida secundaria de la función glandular; susceptibilidad anormal a la colonización endobronquial crónica por un grupo especial de bacterias, como la Pseudomona aeruginosa entre otras (1). Todo mejora con una correcta y adecuada alimentación y la toma de los medicamentos necesarios para el control de esta patología.

La FQ se hereda de manera autosómica recesiva, es decir, un individuo debe tener mutaciones con pérdida de función en ambas copias de su gen CFTR para manifestar la enfermedad (1). Estos alelos o copias mutadas provienen de sus padres, que habitualmente son portadores sanos heterocigotos, es decir, poseen una copia normal de CFTR y una copia mutada. Los portadores sanos no son identificables por síntomas clínicos ni por elevaciones en el test de sudor. Se estima que 1 cada 25 personas de origen nor-europeo es un portador sano. En Chile, esta cifra es probablemente de alrededor de 1 cada 50 personas, o 2\% de la población. Si ambos miembros de una pareja son portadores sanos, tienen un $25 \%$ o 1 en 4 probabilidades de tener un hijo con FQ.Esta cifra permanece igual para cada embarazo (1).

\section{InCIDENCIA POBLACIONAL DE LA FIBROSIS QuístiCA}

La FQ es la enfermedad hereditaria más frecuente en la raza caucásica con consecuencias en la expectativa de vida. En los Estados Unidos y otros países desarrollados la incidencia oscila entre 1:1900 a 1:3700 recién nacidos vivos5, con una frecuencia de portadores del 5\%; por otra parte en asiáticos (6) y afroamericanos la incidencia es más baja (1:32000 recién nacidos vivos y 1:15000 recién nacidos vivos (7) respectivamente).

En cuanto a los hispanos existen pocos estudios que evalúan la frecuencia de la enfermedad (8) encontrándose los datos de Uruguay donde la prevalencia es de 6.9 por cada 100.000 habitantes (9).

\section{ANTECEDENTES HISTÓRICOS DE FQ}

La historia de la fibrosis quística es muy particular, los síntomas de esta enfermedad la confundían o relacionaban con un algún tipo de magias oscuras, por ejemplo, en 1595, el famoso profesor de anatomía y botánica deleiden, Holanda, Peter Pauw (1564-1617), realizó la autopsia de una niña de 11 años que estaba muy delgada debido a una fiebre héctica y tenía pericarditis. En el informe, el doctor anotó: "... se suponía que la niña estaba hechizada ... la niña estaba muy flaca ... el páncreas estaba abultado, cirroso y color blanco brillante..." (1).

En un libro de medicina editado en 1606, escrito por el profesor español Alonso, se puede leer el siguiente párrafo: "...una señora honorable dice que conoce a la gente embrujada, si al rascarles la frente, uno nota después un sabor salado en los dedos..." (1).

O tras antiguas historias, similares a las anteriores, donde el exceso de sal en la frente de los niños, era irremediablemente síntoma de hechizo, encantos, magia, posesión demoníaca, entre otras, se encontraron en otros tantos pueblos de Europa como por ejemplo: Rusia, Polonia, Checoslovaquia, Hungría, Rumania, Italia, Suiza, Austria, etc. Solo cuando Karl Landsteineren 1905 describe la asociación entre meconio espeso en un recién nacido y fibrosis del páncreas, especulando que ambos fenómenos se producen debido a la deficiencia de una enzima (10) encontrando una luz para el diagnóstico de la fibrosis quística.

Con el avance encontrado, en 1936, el pediatra suizo Guido Fanconi fue el primero en usar el término fibrosis quística (FQ) para describir la combinación de insuficiencia pancreática y enfermedad pulmonar crónica en niños (11), pero su reporte se difundió poco por estar escrito en alemán. En 1938, Dorothy Andersen asoció íleo meconial con FQ, notando que las lesiones histológicas en el páncreas eran idénticas en ambas condiciones,y describe este trastorno separado de la enfermedad celíaca (12) El mismo año, Blackfan \& May describen 35 niños con atrofia y fibrosis del páncreas debido a espesamiento de secreciones y dilatación de ductos y acinos (13). En 1943, SydneyFarber reconoce a la FQ como una enfermedad sistémica, y acuña el término "mucoviscidosis" (14). En 1945, Andersen \&Hodges estudian 46 familias de sus pacientes y 56 familias más de la literatura y concluyen quela FQ se hereda en forma autosómica recesiva (15). En 1953: Paul Di Sant' Agnese asigna valor diagnóstico a los electrolitos del sudor (16).gracias a todos estos invetsigadoresen la srespectivas épocas es que hoy tenemos conocimiento claro sobre fibrosis quística.

En 1958 Shwachman y Kulczycki publican su clásica revisión de experiencia con 105 pacientes, el reporte más grande hasta esa fecha. Este paper incluyó una descripción de su score clínico, el que, con algunas actualizaciones, se sigue usando hasta hoy (17). En 1959, 
L. Gibson \& R. Cooke describieron el método de iontoforesis con pilocarpina para estimulación y recolección del sudor, que sigue siendo el estándar hasta la fecha (18). La década de los 1950s se caracterizó por el desarrollo de centros dedicados al estudio y manejo de la FQ, en Europa y Norteamérica. En 1955 se crea la US National CF Research Foundation (posteriormente CF Foundation) y en 1959 la Fundación Canadiense de FQ, y en 1965 se forma en Paris la CF International Association. En los 60, aún la perspectiva para los niños afectados continuaba siendo terrible pues la mayoría moría durante la niñez, después de años de sufrimiento. Recién en 1976, Mitchell-Heggs reporta los primeros 45 pacientes mayores de 12 años, procedentes de 3 hospitales de Londres (19). Sólo en 1979 se pudo disponer de screeningneonatal mediante la utilización de tripsina immunorreactiva (IRT) (20). En 1983 Paul Quinton, portador de $\mathrm{FQ}$, publica que la impermeabilidadal cloro que él había demostrado en las glándulas de sudor, era la base para la elevación de los electrolitos en el sudor de los pacientes con FQ.

Esto se consideró un paso decisivo en la comprensión del defecto básico (21). En 1989, el grupo de LapCheeTsui identifica y clona el gen de la FQ, ubicado en el brazo largo del cromosoma 7, y a la proteína para la cual codifica, la denomina proteína de conductancia transmembrana de la FQ (CFTR, por su sigla en inglés), publicando los hallazgos en un memorable número de la revista Science (22-23). Desde entonces se han identificado más de 1.600 mutaciones, siendo la más frecuente la llamada F508, tanto en Chile como en el resto del mundo. En ese momento, se pensó que rápidamente podría disponerse de terapia génica para la enfermedad, lo que hasta la fecha sigue siendo experimental.

En los últimos 70 años, la FQ ha "mutado" de un trastorno genético poco conocido, usualmente fatal en lactantes y niños, a un complejo trastorno multisistémico, que afecta a muchos niños y adultos. La sobrevida, ha permitido el incremento de condiciones asociadas: osteoporosis, diabetes mellitus, enfermedad hepática, embarazo, infertilidad, entre otros. La demostración que el tratamiento precoz y agresivo mejora el pronóstico obliga a mejorar la precocidad del diagnóstico (tamizaje neonatal) y hacer los mayores esfuerzos en el desarrollo de avances, tanto en las terapias convencionales (farmacológico, nutricional, y demás), como en las más innovadoras (trasplante pulmonar, terapia génica).

\section{ASPECTOS NUTRICIONALES DE LA FQ}

La fibrosis quística es primariamente reconocida por la morbilidad pulmonar asociada a ella, las manifestaciones más tempranas que se anotan en la mayoría de las pacientes están relacionadas con sus alteraciones gastrointestinales y nutricionales (24-25).La ausencia de una función normal de la proteína de membrana CFTR conduce a anomalíasen las glándulas exocrinas que son más notorias en el tracto gastrointestinal por la destrucción de tejido pancreático acinar debido a la obstrucción ductural (26).

La combinación de la actividad enzimática y ausencia de sales biliares conduce a la mala absorción de nutrientes ingeridos especialmente grasas que se manifiestan por la diarrea y el retardo del crecimiento (27).Varios estudios clínicos indican que el estado nutricional desempeñan un papel importante en la progresión de la enfermedad pulmonar (28-29).Estudios longitudinales apuntan a una ventaja de supervivencia para los pacientes con status nutricional optimo (30-30). Los valores de la unción pulmonar en si no parecen repercutir tan fuerte, y por lo tanto, la supervivencia aparece más fuertemente asociadas al crecimiento y en particular a la ganancia en talla (30). Un segundo hallazgo, es que los programas de tratamiento que ponen un mayor énfasis en maximizar la ingesta calórica reportan consistentemente los mejores resultados (30). La insuficiencia pancreática con mala absorción crónica, las infecciones respiratorias recurrentes, la inflamación crónica y el gasto de energía incrementado en combinación con la ingesta óptima son importantes factores determinantes de la destrucción en pacientes con FQ (30).

La mayoría de las personas con FQ tiene una mayor necesidad de calorías, estimada de 120 y $150 \%$ de los requisitos normales. El cuidado nutricional de los pacientes han sido un reto, de hecho, la malnutrición es una complicación muy frecuentes en esta enfermedad y se encuentra en el $20 \%$ de los pacientes.

La detección debe realizarse mediante la combinación de métodos en función de la disponibilidad, ya que existe controversia respecto al protocolo ideal. Igualmente debe llevarse a cabo una valoración detallada y monitorización de la dieta (30).También la determinación de proteínas viscerales puede ayudar a ampliar el diagnostico nutricional, especialmente e situación de reagudización. 
Tabla 1. Según los concesos Europeo y Americano, la clasificación de desnutrición en pacientes adultos con fibrosis quística

\begin{tabular}{lc}
\hline \hline CONSENSO EUROPEO & $\mathrm{IMC}<18.5 \mathrm{Kg} / \mathrm{m}^{2}$ \\
\hline CONSENSO AMERICANO & $\% \mathrm{PI}<90 \% ; \mathrm{IMC}<19 \mathrm{Kg} / \mathrm{m}^{2}$ \\
\hline \hline Tomado de:http://www.nutricion.org
\end{tabular}

Tomado de:http://www nutricion org

Tabla 2. Recomendaciones dietéticas para pacientes con fibrosis quística.

\begin{tabular}{lc}
\hline \hline COMPONENTE & CANTIDAD \\
\hline Energía & $\begin{array}{c}120-150 \% \text { de las calorías } \\
\text { recomendadas para personas sanas } \\
\text { de su misma edad, sexo y } \\
\text { composición corporal. }\end{array}$ \\
Hidratos de Carbono & $40-50 \%$ VCT \\
Proteínas & $15-20 \%$ VCT \\
& $35-40 \%$ VCT \\
Lipidos & $<10 \%$ AGS \\
& $<10 \%$ AGP \\
Fibra & $<1 \%$ AGTrans \\
\hline \hline Tomado de: http://www.nutricion.org & AGM: el resto \\
VCT: Valor calórica total. AGs: acido grasos saturados. AGP: Acidos grasos poliinsaturados, \\
AGM: acidos grasos moniinsaturados; DHA: Acidos docosahexaenoico; EPA: Acido \\
eicosapentaenoico; GLA: Acidos gammalinolenico.
\end{tabular}

Tabla 3. Recomendaciones alimenticias para el aumento de ingesta calóricas y proteicas en pacientes con fibrosis quística.

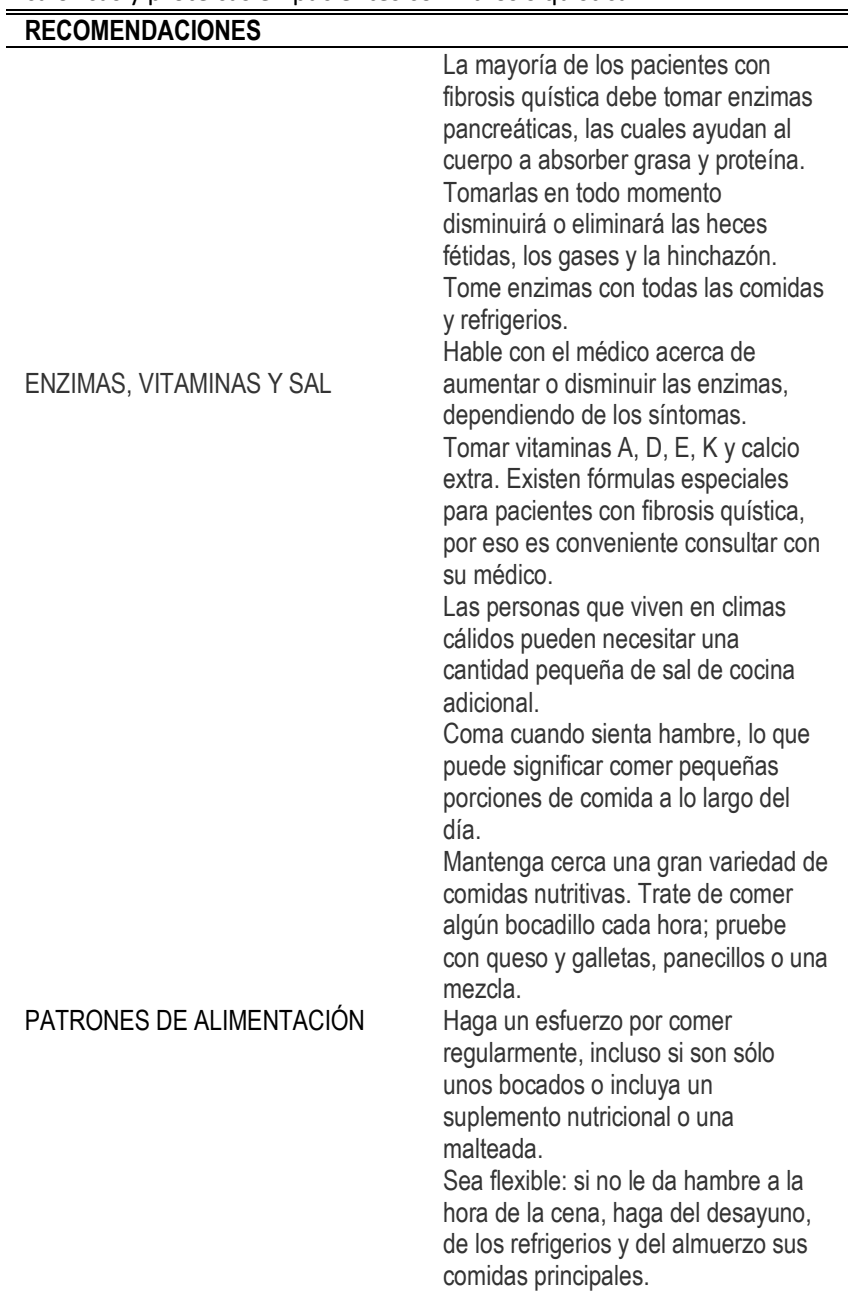

\begin{tabular}{|c|c|}
\hline $\begin{array}{l}\text { OBTENER MÁS CALORÍAS Y } \\
\text { PROTEÍNAS }\end{array}$ & 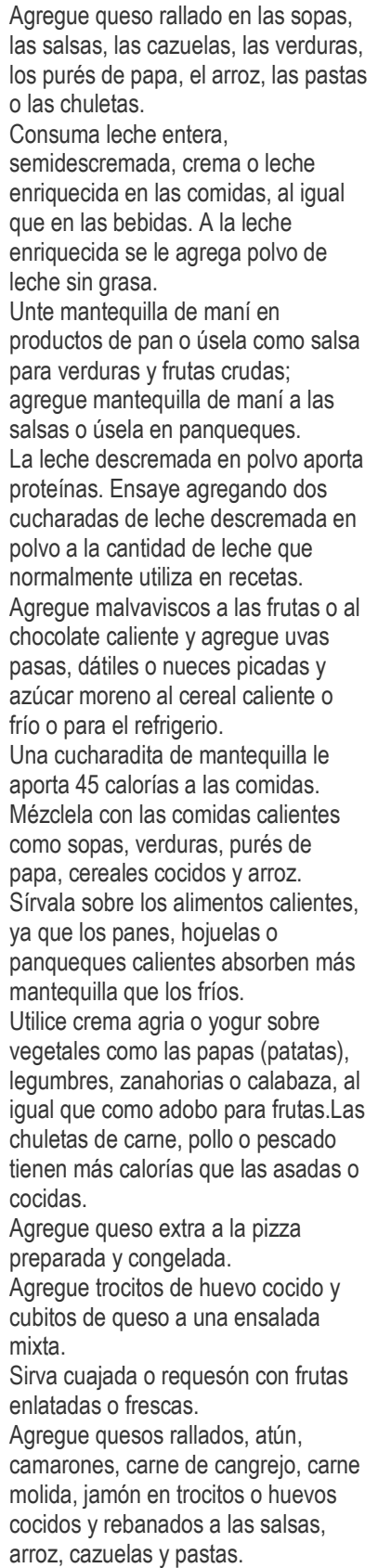 \\
\hline
\end{tabular}

Tomado de :http://loyolauniversity.adam.com 
existe un tratamiento estandarizado sino que, más bien, es el especialista quien determina en cada momento cuál es el tratamiento más adecuado (30). Aun así, los tratamientos y disciplinas que actualmente se aplican son:

- $\quad$ Ejercicios de fisioterapia respiratoria. Precedidos de la inhalación de distintos medicamentos para la fluidificación de la mucosidad.

- Tratamientos antibióticos, por vía oral, intravenosos o inhalados.

- Enzimas pancreáticas en cada comida, administradas por vía oral en forma de cápsulas.

- En los casos en los que se desarrolla diabetes, el tratamiento propio de esta enfermedad.

- Complementos vitamínicos.

- Complementos calóricos, además de una dieta adecuada.

- Bebidas isotónicas para prevenir deshidrataciones.

- Es aconsejable la realización de algún ejercicio físico que ayude a mejorar la capacidad pulmonar.

- $\quad$ En los casos de degeneración irreversible y grave de la capacidad pulmonar, el transplante de pulmón es la única vía de solución posible.

\section{CONCLUSIONES}

Después de haber analizado la información presente en el documento se puede concluir que la fibrosis quística es una de las patologías de mayor incidencia en poblaciones caucásicas, esta es de naturaleza autosómica recesiva y su expresión se relaciona con factores químicos como con factores físicos, de los cuales destacamos la sobreproducción de secreciones mucosas a nivel pulmonar y gástrico.

La sintomatología de la Fibrosis Quística varía en función de la edad del individuo, el grado en que se ven afectados órganos específicos, la terapéutica instituida previamente, y los tipos de infecciones asociadas.

La mayoría de las personas con FQ tiene una mayor necesidad de calorías por los que el cuidado nutricional de los pacientes ha sido un reto, de hecho, la malnutrición es una complicación muy frecuente en esta enfermedad y se encuentra en el $20 \%$ de los pacientes.

En cuanto al tratamiento, no hay un proceso o esquema particular para algunas de las variantes de la Fibrosis Quística, de hecho el esquema estipulado para cada una de estas variantes se encamina hacia mejorar la calidad de vida del paciente. Este esquema varía dependiendo de las características físicas, nutricionales y genética del paciente.

\section{REFERENCIAS BIBLIOGRÁFICAS}

1. Vásquez C, Aristizábal R, Daza W. Fibrosis Quística En Colombia. Disponible en web: http://www.neumologia-pediatrica.cl

2. Riordan JR, Rommens JM, Kerem B, Alon $N$, Rozmahel R, Grzelczak Z,.Identification of thecystic fibrosis gene: cloning and characterization of complementary DNA. Science 1989; 245: 1066-73.

3. Collazo T, Piloto $Y$, Clark $Y$, Bofill AM, Gomez $M$, Hernandez $Y$. Deteccion de mutaciones en pacientes cubanos con fibrosis quística. Biotecnologia aplicada 2008;25:345-349.

4. Cystic Fibrosis GeneticAnalysisConsortium. Worldwidesurvey of the DF508 mutation. ReportFromtheCystic Fibrosis GeneticAnalysisConsortium. Am J HumGenet 1990;47: 354-9.

5. Welsh MJ, Ramsey BW, Accurso F. Cutting G. Cystic fibrosis. In: scriver CR, Beaudet AL, Sly WS, Valle $D$, editors. Themetabolic and molecular basis of inheriteddiseases, 8th ed. New York: Mcgraw - Hill; 2001 paginas $5121-88$.

6. Imalzumi Y. Incidence and mortalityrates of cystic fibrosis in japan, 1969-1992. Am JmedGenet 1995;58:161 - 168.

7. Hamosh A. Firtz - Simmons SC, Macek M Jr. Knowles MR, Rosentein BJ, Cutting GR. Comparison of theclinicalmanifestations of cystic fibrosis in blackand White patients. J pediatr 1998; 132: 255 - 59.

8. Macri CN, de Gentile AS, Manterola A. et al. Epidemiology of cystic fibrosis in LatinAmerica: preliminarycommunication. PediatrPulmonol 1991; 10:249-53.

9. Grebbe TA, Doane WWW, Richter SF, Seltzer WK, Jain KD. A rationalapproachtocystic fibrosis mutationanalysis in Hispanics: replytoArzimanoglou et al. Am J HumGenet 1996;59: 269-72.

10. Di Santagnese PA, Darling RC, Perera GA, Shea E. Abnormmalelectrolytecomposition of sweat in cystic fibrosis of the páncreas; clinicalsignificance and relationshiptothedisease. Pediatrics. 1953; 12: 54963.

11. Shwachman H, Kulczycki LL. Long- termstudy of onehundredfivepatientswithcystic fibrosis; studiesmadeover a five - tofourteen- yearperiod. AMA J DisChild 1958; 96: 6- 15.

12. Gibson LE, Cooke RE. A test forconcentration of electrolytes in sweat in cystic fibrosis of the páncreas utilizingpilocarpinebyiontophoresis. Pediatrics 1959; 23: 545-9. 
13. MitchellHeggs P. Mearns M. Batten JC. Cystic fibrosis in adolescents and adults. Q J Med 1976; 45: 479504.

14. Crossley JR, Elliott RB, Smith PA. Dried- blood spot screeningforcystic fibrosis in thenewborn. Lancet 1979: I: 472- 4.

15. Quinton PM. Chlorideimpermeability in cystic fibrosis. Nature 1983: 301: 421-2.

16. Kerem B, Rommens JM, Buchanan JA, Markiewicz D, Cox TK. Chakravarti A, Buchwald M. Tsui LC. Identification of thecystic fibrosis gene: geneticanalysis. Science 1989; 245: 1073 -880.

17. Rommens JM. Lannuzzi MC, Kerem B. Drumm ML, Melmer G, Dean M, Rozmahel R, Cole JL, Kennedy D, Hidaka $\mathrm{N}$, et al. Identification of thecystic fibrosis gene: choromosomewalking and jumping. Science 1989; 245: $1059-65$.

18. Davies JC, Alton EWFW, Bush A. Cystic fibrosis. BMJ 2007; 335: 1255-9

19. Davis PB, Drumm M, Konstan MW. Cystic fibrosis. Am J RespCritCareMed 1996; 154: 1229-56

20. Durie PR. Thepathophysiology of thepancreaticdefect in cystic fibrosis. Acta PaedScanSupp 1989; 363: 41-4

21. Walkowiak J, Lisowska A, Blaszczynski M. Thechangingface of theexocrinepancreas in cystic fibrosis: pancreaticsufficiency, pancreatitis and genotype. Eur J GastrHepatol 2008; 20: 157-60.

22. Kraemer R, Rudeberg A, Hadorn B, Rossi E. Relativeunderweight in cystic fibrosis and itsprognosticvalue. Acta PaedScan 1978; 67: 33-7

23. Nir M, Lanng S, Johansen HK, Koch C. Longtermsurvival and nutritional data in patientswithcystic fibrosis treated in a Danish centre. Thorax 1996; 51: 1023-7

24. Corey M, McLaughlin FJ, Williams M, Levison H. A comparison of survival, growth and pulmonaryfunction in patientswithcystic fibrosis in Boston and Toronto. J ClinEpidemiol 1988; 41: 58391

25. Lai HC, Corey M, FitzSimmons S, Kosorok MR, Farrell PM. Comparison of growth status of patientswithcystic fibrosis betweentheUnitedStates and Canada. Am J ClinNutrit 1999; 69: 531-8.

26. Beker LT, Russek-Cohen E, Fink RJ. Stature as a prognostic factor in cystic fibrosis survival. J Am DieteticAssoc 2001; 101: 438-4

27. Corey M, Gaskin K, Durie P, Levison H, Forstner G. Improved prognosis in $\mathrm{CF}$ patientswith normal fatabsorption. J PedGastroenterNutr 1984; 3 Suppl 1:S99-105.

28. Milla C. Nutrition and LungDisease in Cystic Fibrosis.
ClinChestMed 2007;28:12

29. Consideraciones nutricionales para la fibrosis quística. Disponible en la web: http://loyolauniversity.adam.com

30. Fibrosis Quistica. Tomado: 29-nov.-13. Disponible en la web: http://www.dmedicina.com/enfermedades/respirato rias/fibrosis-quistica.
Las opiniones que contenga este artículo son de su(s) autor(es) y no necesariamente representan la opinión oficial de la Universidad Simón Bolivar, la Revista Ciencia e Innovación en Salud o de sus autoridades. 